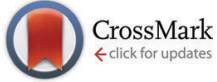

Cite this: Phys. Chem. Chem. Phys., 2015, 17, 8983

Received 16th January 2015 , Accepted 21st February 2015 DOI: $10.1039 / \mathrm{c} 5 \mathrm{cp} 00289 \mathrm{c}$

www.rsc.org/pccp

\section{The impact of steam on the electronic structure of the selective propane oxidation catalyst MoVTeNb oxide (orthorhombic M1 phase) $\dagger$}

\author{
Christian Heine, ${ }^{a}$ Michael Hävecker, ${ }^{a b}$ Annette Trunschke, ${ }^{a}$ Robert Schlögl ${ }^{a}$ and \\ Maik Eichelbaum*ac
}

\begin{abstract}
The selective propane oxidation catalyst MoVTeNb oxide M1 was investigated by microwave conductivity, synchrotron X-ray photoelectron, soft X-ray absorption and resonant photoelectron spectroscopy under reaction conditions to identify the influence of steam on the electronic bulk and surface properties. Steam significantly increases both the conversion of propane and the selectivity to the target product acrylic acid. The increased catalytic performance comes along with a decreased conductivity, a modification of the surface chemical and electronic structure with an enrichment of covalently bonded $\mathrm{V}^{5+}$ species to the extent of $\mathrm{Mo}^{6+}$, a decreased work function and hence polarity of the surface and a modified valence band structure. The higher degree of covalency in metal oxide bonds affects the mobility of the free charge carriers, and hence explains the decrease of the conductivity with steam. Furthermore we could prove that a subsurface space charge region depleted in electrons and thus an upward bending of the electronic band structure are induced by the reaction mixture, which is however not dependent on the steam content.
\end{abstract}

\section{Introduction}

Acrylic acid with an annual global production of 4.7 million tonnes (2012) is an important intermediate in the chemical industry for the production of polyacrylates and methacrylate esters for diverse applications, e.g. for superabsorbers. It is currently mostly manufactured with a yield of up to $80 \%$ by a two step heterogeneous catalytic process starting with the oxidation of propylene to acrolein followed by its oxidation to acrylic acid. ${ }^{1}$ Propylene is produced by steam cracking of the naphtha distillation fraction of crude oil. The endothermic steam cracking process is highly energy-consuming due to the high temperatures needed (up to $850{ }^{\circ} \mathrm{C}$ ) and the necessary steam generation. The upcoming scarcity of crude oil resources and the corresponding expected price increase are serious factors that would reduce the cost effectiveness of the current acrylic acid manufacture process. A competitive alternative could be the direct oxidation of propane from natural gas. The so-far most promising catalyst for direct acrylic acid production from propane with a

\footnotetext{
${ }^{a}$ Department of Inorganic Chemistry, Fritz-Haber-Institut der

Max-Planck-Gesellschaft, Faradayweg 4-6, 14195 Berlin, Germany.

E-mail: me@fhi-berlin.mpg.de; Fax: +49 (0)30 84134405; Tel: +49 (o)30 84134566

${ }^{b}$ Solar Energy Research, Helmholtz-Zentrum Berlin/BESSY II,

Albert-Einstein-Straße 15, 12489 Berlin, Germany

${ }^{c}$ BasCat, UniCat BASF JointLab, TU Berlin, Marchstraße 6, 10587 Berlin, Germany

$\dagger$ Electronic supplementary information (ESI) available: O1s and Te3d X-ray photoelectron spectra of MoVTeNb oxide. See DOI: 10.1039/c5cp00289c
}

current acrylic acid yield of about $50 \%$ is the orthorhombic MoVTeNb oxide M1 phase (ICSD No. 5509; in our publication simply abbreviated as “M1"). ${ }^{2-5} \mathrm{M} 1$ is a multimetal mixed oxide that can be synthesized with high phase purity by powder X-ray diffractometry (XRD). It should be noted that XRD phase purity does not exclude inhomogeneities such as cation disorder or dynamic overlayers terminating the crystal surface, which can be different from the bulk structure. ${ }^{6,7}$ The (ideal) crystal structure of M1 provides hexagonal and heptagonal channels perpendicular to the (001) basal plane partially occupied by tellurium. The (001) basal plane is formed by corner-linked $\mathrm{MO}_{6}$ octahedra $(\mathrm{M}=$ molybdenum or vanadium) building a six and seven membered ring structure. $\mathrm{MO}_{7}(\mathrm{M}=$ mainly niobium $)$ pentagonal bipyramids connect the ring structures by sharing edges with the octahedra. Along the $c$-direction the (001) basal planes are linked by oxygen atoms.

In the oxidation of propane with oxygen on M1, water (i.e. steam) plays a peculiar role in the selective oxidation reaction. In dry feed containing only propane and oxygen, propylene formed by oxidative dehydrogenation $(\mathrm{ODH})$ is the main product (besides $\mathrm{CO}$ and $\mathrm{CO}_{2}$ ), while the selectivity to acrylic acid is rather low. ${ }^{8}$ However, the selectivity to acrylic acid is substantially increased up to $60 \%$ if steam is added to the reaction feed. ${ }^{8,9}$ It was proposed that water increases the desorption rate of acrylic acid on the catalyst surface preventing its over-oxidation (e.g. by transforming surface carboxylates into acrylic acid), but it also changes the nature of the active sites. ${ }^{8}$ In general, recent studies indicate that the 
bulk phase of M1 provides the framework of the active surface which is formed only under reaction conditions. ${ }^{7,9,10}$ While during the catalytic reaction the bulk structure of M1 is unaffected, ${ }^{8}$ the surface structure changes depending on the composition of the gas phase. Near-ambient pressure X-ray photoelectron spectroscopy (NAP-XPS) exhibited that the average oxidation states of vanadium and molybdenum are substantially increased at the surface. ${ }^{7,10}$ Molybdenum is only found in the formal oxidation state of $6+$ on the surface, while in the bulk a ratio of molybdenum(v) and (vI) is needed to form the crystal structure. ${ }^{4}$ Vanadium is a redox active element in M1 with a gas phase-dependent oxidation state, as known from a careful analysis of the vanadium $2 \mathrm{p}_{3 / 2}$ core level. The addition of water to the propane feed, for triggering the production of acrylic acid, induces an increase in the $\mathrm{V}^{5+}$ concentration $^{7,10}$ which is suggested to be essential for the alkane activation step. ${ }^{10,11}$ The $\mathrm{V}^{4+}$ concentration is unaffected by the water content of the propane feed ${ }^{7,8,10}$ supporting the assumption of the unique role of $\mathrm{V}^{5+}$ in the acrylic acid formation. Moreover, the tellurium content increases while the molybdenum content decreases with steam. ${ }^{7,10}$ That is why it has been suggested that tellurium is also part of the active site. ${ }^{9}$

In catalysis chemical modifications on the surface of the catalyst are in general interpreted in terms of a localized molecular model. However, regarding the crystalline, n-type semiconducting MoVTeNb oxide $\mathrm{M}^{12}$ the collective solid state properties such as the valence and conduction band structures, work function and electrical conductivity also have to be taken into account. It was proven recently by microwave conductivity, which is a contactless technique allowing the investigation of the electrical properties of catalysts in a fixed-bed reactor without electrode contact resistance limitations, ${ }^{13}$ and NAP-XPS studies that M1 acts as a semiconducting gas sensor by variation of the reaction conditions. ${ }^{12}$ The conductivity, work function and valence band onset are adjusted reversibly to the applied chemical potential of the gas phase. Consequently, an active charge transfer between the bulk and the gas phase (or adsorbates) was proven indicating the relevance of the semiconducting properties of the bulk phase in controlling the catalytically active surface structure.

In our contribution we want to identify how steam modifies the active surface layer catalyzing the selective oxidation of propane to acrylic acid, and if it also changes the collective electronic properties of the M1 phase. The latter was investigated by microwave conductivity measurements under dry and wet feed conditions at ambient pressure. The results are compared and interpreted with surface sensitive synchrotron-based in situ NAP-XPS and near-edge X-ray absorption fine structure (NEXAFS) investigations at 0.25 mbar under dry and wet propane oxidation conditions to identify structure-function relationships between the local electronic surface structure of M1, and its collective electronic and catalytic properties. Core level spectra of vanadium $2 \mathrm{p}_{3 / 2}$, molybdenum $3 \mathrm{~d}$, tellurium $3 \mathrm{~d}$ and oxygen 1 s, valence band spectra and the work function were analyzed. Moreover, the contribution of vanadium to the electronic structure of the valence band and gap states was investigated in detail by resonant photoelectron spectroscopy (resPES) at the vanadium $2 p$ threshold (vanadium $\mathrm{L}_{2,3}$-edge).

\section{Experimental}

\subsection{Sample}

The MoVTeNb oxide M1 phase (internal ID.: 10790) was prepared according to a precipitation procedure described previously. ${ }^{12}$ Relative metal contents of 65 at\% Mo, 6 at\% Te, 19 at\% V and 10 at $\% \mathrm{Nb}$ were determined by X-ray fluorescence analysis. The surface area of the sample is $9.2 \mathrm{~m}^{2} \mathrm{~g}^{-1}$ as measured by BrunauerEmmett-Teller (BET) nitrogen adsorption. The Rietveld analysis of the X-ray powder diffraction (XRD) pattern proved the phase purity of the used M1 powder. It has also been proven by in situ XRD that the bulk phase is stable under all applied reaction conditions. ${ }^{8}$

\subsection{Microwave conductivity measurements}

The electrical conductivity of M1 under reaction conditions was measured by the microwave cavity perturbation technique. In the depolarization regime, the semiconducting sample located in a microwave cavity causes a perturbation of the resonant electromagnetic field decreasing its quality factor $Q$ and resonance frequency $\omega_{0} \cdot{ }^{14}$ The dielectric function (permittivity) of the sample $\left(\varepsilon=\varepsilon_{1}+i \varepsilon_{2}\right)$ can be computed by using the quality factor/ resonance frequency of the perturbed $\left(Q_{\mathrm{s}} / \omega_{\mathrm{s}}\right)$ and unperturbed cavities $\left(Q_{0} / \omega_{0}\right){ }^{15}$

$$
\begin{gathered}
A\left(\varepsilon_{1}-1\right) \frac{V_{\mathrm{s}}}{V_{\mathrm{c}}}=\frac{\omega_{0}-\omega_{\mathrm{s}}}{\omega_{0}} \\
B \varepsilon_{2} \frac{V_{\mathrm{s}}}{V_{\mathrm{c}}}=1 / Q_{\mathrm{s}}-1 / Q_{0}
\end{gathered}
$$

In eqn (1) and (2) $V_{\mathrm{s}} / V_{\mathrm{c}}$ is the sample/cavity volume ratio. $A$ and $B$ are proportionality factors known from calibration standards. ${ }^{13,15}$ In the present study the sample was polycrystalline and therefore an effective permittivity is obtained, which still depends on the packing fraction, particle size and shape etc. To determine the single crystal (bulk) permittivity the effective values have to be transformed, by an effective medium theory, into the bulk values. We used the Landau-Lifshitz-Looyenga effective medium model because it was found to be suitable for nano- and microcrystalline powders. ${ }^{16,17}$ The single crystal conductivity $\sigma$ of the sample can be finally calculated with the imaginary part of the single crystal permittivity $\varepsilon_{2}$ :

$$
\sigma=\varepsilon_{0} \omega_{\mathrm{s}} \varepsilon_{2}
$$

Details about the in situ microwave conductivity setup were reported previously. ${ }^{13,18}$ The measurement was performed in the $\mathrm{TM}_{110}$ mode of a cylindrical cavity at a resonance frequency of about 9.2 GHz. In the experiment the total gas flow controlled by

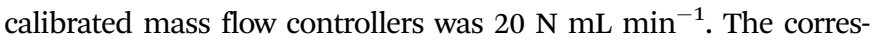
ponding gas hourly space velocity was $13333 \mathrm{~N} \mathrm{~mL}(\mathrm{~g} \mathrm{~h})^{-1}$ (contact time: $0.27 \mathrm{~s} \mathrm{~g} \mathrm{~N} \mathrm{~mL}^{-1}$ ) with a catalyst mass of $90 \mathrm{mg}$ and a bed length of $10.6 \mathrm{~mm}$ in a quartz tube reactor with a $3 \mathrm{~mm}$ inner diameter. A particle sieve fraction between 100 and $200 \mu \mathrm{m}$ was used for the experiment. The measurement was performed under wet and dry feed conditions containing 3 vol\% propane, 6 vol\% oxygen and $5 \mathrm{vol} \%$ steam for the wet feed with residual $\mathrm{N}_{2}$ gas at $350{ }^{\circ} \mathrm{C}$. 


\subsection{Near-ambient pressure X-ray photoelectron and X-ray} absorption spectroscopy

X-ray photoelectron spectroscopy (XPS), resonant photoelectron spectroscopy (resPES) and near edge X-ray absorption fine structure (NEXAFS) studies were performed at the ISISS (Innovative Station for In Situ Spectroscopy) beamline at the synchrotron facility BESSY II in Berlin, Germany. Monochromatic light was used to record high resolution in situ XP and NEXAFS spectra. A detailed description of the differentially pumped hemispheric electron analyzer and the in situ vacuum chamber was reported before. ${ }^{19,20}$ For the measurements the sample powder was pressed into a self-supporting pellet of $8 \mathrm{~mm}$ diameter $(1$ ton pressing pressure). The experiment was performed at $25 \mathrm{~Pa}$ and $400{ }^{\circ} \mathrm{C}$ (heating rate $5 \mathrm{~K} \mathrm{~min}^{-1}$ ). The reaction feed contained $1 \mathrm{~N} \mathrm{ml} \mathrm{min}{ }^{-1}$ propane, $2 \mathrm{~N} \mathrm{ml} \mathrm{min}{ }^{-1}$ oxygen and $3 \mathrm{~N} \mathrm{ml} \min ^{-1}$ helium for the

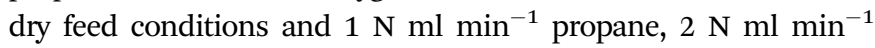
oxygen and $3 \mathrm{~N} \mathrm{ml} \mathrm{min}{ }^{-1}$ steam for the wet feed conditions. The experiment was started in the wet feed, followed by dry and finally changed again to wet feed conditions.

NEXAFS experiments were performed in the total electron yield (TEY) mode and in the Auger-Meitner electron yield (AMEY) mode. The photon energy was varied from 505 to $560 \mathrm{eV}$ by continuously moving the monochromator at a constant velocity of $217 \mathrm{meV} \mathrm{s}^{-1}$ to record the vanadium L-edges and the oxygen K-edge. The exit slit setting was $111 \mu \mathrm{m}$. The energy positions of every raw data points were read back from the monochromator control. The generated electrons are accelerated to a Faraday cup by the electric field (applied voltage) and the photocurrent is measured. The AMEY data were acquired using an electron spectrometer setting the kinetic energy at $385 \mathrm{eV}$ (pass energy $20 \mathrm{eV}$ ) to suppress contributions of the gas phase to the AMEY, in particular from molecular oxygen. Details about the methodology to suppress gas phase contributions in the NEXAFS signal were reported before. ${ }^{21}$

Core level spectra of O1s, V2p, Mo3d and Te3d were performed at a pass energy of $20 \mathrm{eV}$ with constant kinetic electron energies of 150 and $650 \mathrm{eV}$, respectively, corresponding to inelastic mean free paths (IMFPs) of $0.6 \mathrm{~nm}$ (surface sensitive) and $1.6 \mathrm{~nm}$ ("bulk" sensitive). The IMFP was calculated for $\mathrm{MoO}_{3}$ using the model of Tanuma et $a .^{22}$ Normalized core level intensities were evaluated after subtraction of a Shirley type background (BG) taking into account the photon energy dependence of the atomic subshell photoionization cross sections, using CASA data analysis software (Neil Farley, www.casaxps.com). Valence spectra and secondary electron cutoffs of the photoelectron spectra were recorded at a photon energy of $100 \mathrm{eV}$. A voltage of $-18 \mathrm{~V}$ was applied between the sample and the photoelectron detector to measure the cutoff of the photoelectron spectrum. The electron escape vector was perpendicular to the surface of the pellet to avoid a distortion of the electron trajectories by the electric field. ${ }^{23}$ In the case of the resPES measurements at the vanadium $2 p$ threshold, the valence band spectra were recorded at characteristic points of the vanadium $\mathrm{L}_{2,3}$-edge. The monochromator was operated in a higher diffraction order suppression mode $(c=1.4)$. Further details about the measurement are discussed in the results section.

\section{Results and discussion}

\subsection{Microwave conductivity measurements}

Microwave conductivity measurements of M1 in a fixed-bed reactor under propane oxidation conditions were performed at $350{ }^{\circ} \mathrm{C}$ and with a contact time of $0.27 \mathrm{~s} \mathrm{~g} \mathrm{~N} \mathrm{ml}^{-1}$ to achieve a low propane conversion of $<5 \%$ (differential conditions) for better comparison with the low pressure in situ XPS results (propane conversion of less than 1\%) and to avoid concentration (and conductivity) gradients across the sample. Fig. 1a shows the microwave conductivity of M1 obtained under dry and wet feed conditions, while the simultaneously measured catalytic performance is plotted in Fig. 1b. In the wet feed containing $3 \%$ propane, $6 \%$ oxygen and $5 \%$ steam the selectivity to acrylic acid is significantly increased from $10 \%$ to $30 \%$, as compared to that under the conditions without steam. The propane conversion increases also from $3 \%$ to $4 \%$. In contrast, the selectivities to propylene (from $80 \%$ to $60 \%$ ) and to the undesired total oxidation products $\mathrm{CO}$ and $\mathrm{CO}_{2}$ (from $\mathrm{ca}$. $7 \%$ to $4 \%$ ) decrease with steam. These reversible and stable trends and even the absolute values are basically in agreement with the catalytic data reported recently for similar conversions in a comprehensive reactivity study of $\mathrm{M} 1$ tested in a broad parameter field. ${ }^{8}$ Based on these results the effect of water was interpreted in terms of the suppression of a decomposition of acrylic acid to $\mathrm{CO}$ and $\mathrm{CO}_{2}$, explaining the increased selectivity to acrylic acid and the decreased selectivity to $\mathrm{CO}_{x}$. However, the increased conversion has to be explained by an additional effect of water on the nature of the active sites with increased turn-over frequencies and
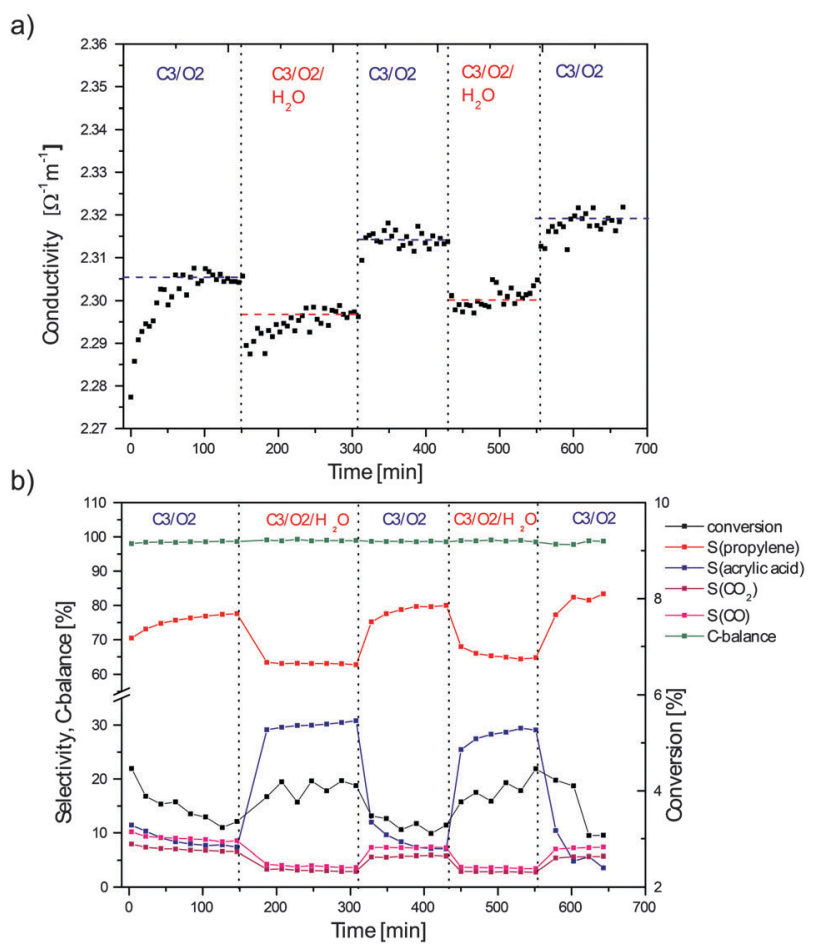

Fig. 1 (a) Microwave conductivity of M1 under dry and wet propane feed conditions at $350{ }^{\circ} \mathrm{C}$ and (b) simultaneously measured catalytic data. 
decreased activation energies. The investigation of this latter effect is the focus of the present study.

Upon reaching the final temperature of $350{ }^{\circ} \mathrm{C}$ (time point " 0 " in Fig. 1a) the conductivity increased until it stabilized at a value of about $2.305 \Omega^{-1} \mathrm{~m}^{-1}$ after 1.5 hours. This value is in the range of the conductivity measured for pure silicon at $350{ }^{\circ} \mathrm{C}$, which is much smaller than that for doped silicon samples, ${ }^{24}$ but orders of magnitude higher than determined for the selective $n$-butane oxidation catalyst vanadyl pyrophosphate (VPO). ${ }^{25}$ The wet feed conditions were applied after the conductivity value remained constant for 1 hour. The feed change induced an immediate decrease followed by a slow increase in conductivity to a constant value still lower than the orignal conductivity in the dry feed. After applying the dry gas mixture for the second time the conductivity increased instantaneously to a constant value slightly above the value measured under the first dry conditions. The repeated change to wet and then again dry feed gave qualitatively the same results as obtained before with a generally lower conductivity in the presence of steam.

In a previous study probing the microwave conductivity of M1 as a function of dry alkane oxidation feeds (with different alkanes, ethane, propane, $n$-butane and different alkane/oxygen ratios), the conductivity decreased while going from reducing to oxidizing conditions indicating the n-type semiconductivity of M1 with electrons as majority charge carriers. ${ }^{12}$ In this study the work function, the valence band and core levels of M1 were additionally investigated by NAP-XPS. As a result, the work function of M1 increased from reducing to oxidizing gas atmospheres. The analysis of the peak area and shape of the core level spectra exhibited no significant changes of the surface elemental composition, but a slight change in the vanadium oxidation state. Furthermore, the core and valence levels shifted to lower energies in oxidizing and to higher energies in reducing feeds. This can be explained by a typical semiconductor response of a gas sensor, where the Fermi level of the solid phase is pinned to the surface state energy modified by the adsorbates. ${ }^{26}$ As a consequence, the conduction and valence bands and all core levels are bent towards the surface, as indicated by the consistent shift to lower binding energies, because charge carriers are transferred between the surface (adsorbates) and the bulk phase, while a subsurface space charge region and an oppositely charged surface layer are formed. The electrical conductivity $\sigma(x)$ depends on the spatially varying energy interval between the conduction band onset $E_{\mathrm{C}}$ and the Fermi energy $E_{\mathrm{F}}$ in the space charge region ${ }^{26}$ after

$$
\sigma(x) \approx e n_{\mathrm{e}}(x) \mu_{\mathrm{e}}, n_{\mathrm{e}}=N_{\text {eff }}^{C} \exp \left[-\frac{E_{\mathrm{C}}(x)-E_{\mathrm{F}}}{k T}\right],
$$

where $e$ is the elementary charge, $n_{\mathrm{e}}$ the free electron density, $\mu_{\mathrm{e}}$ the free electron mobility, $N_{\text {eff }}^{\mathrm{C}}$ the effective conduction band density of states, $k$ the Boltzmann constant and $T$ the temperature. Consequently, the electrical conductivity is directly influenced by the degree of band bending, modifying the gap between $E_{\mathrm{C}}$ and $E_{\mathrm{F}}$. This is the physical explanation for the correlation found between band bending (i.e. core and valence level shifts), work function and microwave conductivity with all being dependent on the density of free charge carriers. If the decreased conductivity of M1 in the steam containing propane oxidation feed, as observed in our present study, can be explained by a steam-modified band bending as well, and hence by a modification of the charge carrier density in the bulk phase, is the rationale for the investigation of the catalyst by NAP-XPS in dry and wet feeds, which will be reported in the next sections.

\subsection{Core level spectra}

We analyzed oxygen $1 \mathrm{~s}$, vanadium $2 \mathrm{p}$, molybdenum $3 \mathrm{~d}$ and tellurium 3d core level spectra of M1 under dry and wet feed conditions by NAP-XPS. Two questions will be addressed by this study: (1) can we prove a bending of bands under selective propane oxidation conditions and hence the existence of a space charge region extending from the surface into the bulk by measuring the binding energy of photoelectrons in different depths of the catalyst? (2) If band bending is observed, does it depend on steam, which would allow a correlation between the selectivity to acrylic acid, showing a strong dependence on steam content, and the semiconducting response of the catalyst? The core levels were recorded in a surface sensitive (photoelectron kinetic energy: $150 \mathrm{eV}$; inelastic mean free path (IMFP): $0.6 \mathrm{~nm}$, meaning that $63 \%$ of the photoelectrons were released within this depth) and "bulk" sensitive ( $650 \mathrm{eV}$, IMFP: $1.6 \mathrm{~nm}$ ) mode to study the extension of the space charge region from the surface into the bulk and to discriminate between upward (i.e. formation of a depletion layer) and downward band bending (accumulation layer). A steam-dependent band bending can be proven if the core level binding energies are systematically shifted between dry and wet reaction feeds. In Fig. 2a the surface-sensitively recorded O1s and V2p spectra are shown. It is obvious that the maximum of the O1s peak appears at the same binding energy position independent of the applied feed conditions. Moreover, difference spectra between measurements in dry and wet feed gave no hint for a specific contribution to the O1s spectrum that is directly caused by the addition of steam to the gas phase (Fig. S1, ESI $\dagger$ ).

Since the satellite of the $\mathrm{V}^{4+}$ contribution to the $\mathrm{V} 2 \mathrm{p}_{3 / 2}$ spectrum overlaps with the V2 $\mathrm{p}_{1 / 2}$ peak (Fig. 2a), only the undistorted V2 $\mathrm{p}_{3 / 2}$ core level was used to analyze chemical shifts. The $\mathrm{V} 2 \mathrm{p}_{3 / 2}$ spectra are shown in detail in Fig. 2b. The spectra were fitted with two Gaussian-Lorentz peaks corresponding to $\mathrm{V}^{4+}$ and $\mathrm{V}^{5+}$ components. ${ }^{7,8,10}$ Though a clear increase of the $\mathrm{V}^{5+}$ contribution is observed with steam, the absolute binding energies are for both oxidation state components independent of the feed.

The Mo3d doublet is depicted in Fig. 2c. Independent of the applied feed conditions this core level can be fitted with just one component ${ }^{7,8,10}$ corresponding to $\mathrm{Mo}^{6+} \cdot{ }^{27}$ Again, the maximum of this spectrum is not shifted between dry and wet propane oxidation feeds, which is in agreement with the other core level spectra, but in apparent contradiction to the previous assumption correlating the conductivity change with a gas phase dependent modification of the band bending, as indicated in Fig. 4.

In Fig. 2d, e and $\mathrm{f}$ the bulk sensitively recorded $\mathrm{O} 1 \mathrm{~s}, \mathrm{~V} 2 \mathrm{p}_{3 / 2}$ and Mo3d core level spectra are shown and compared by the 
a)

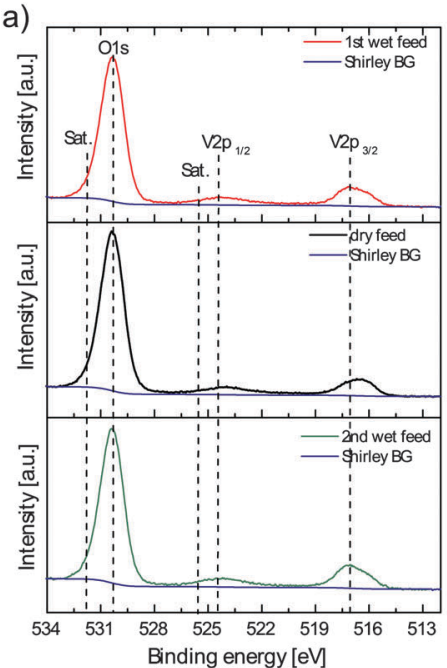

d)

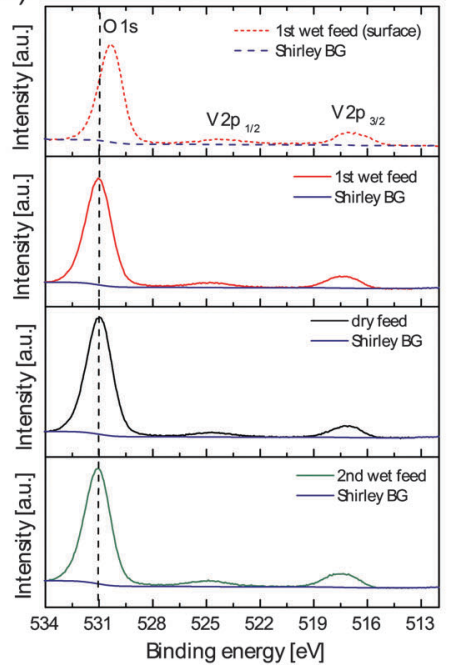

b)

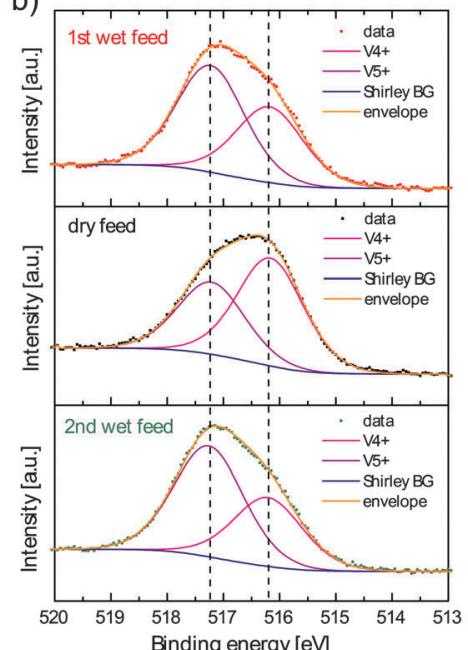

e)

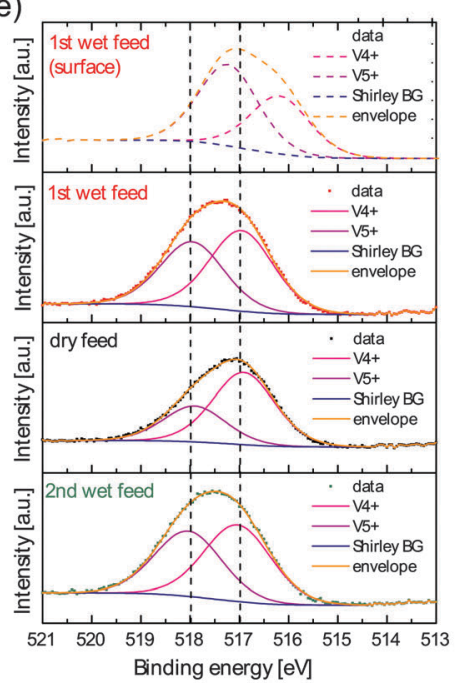

c)

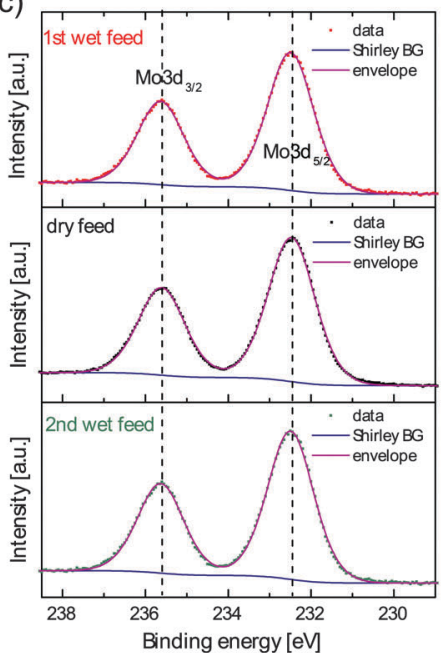

f)

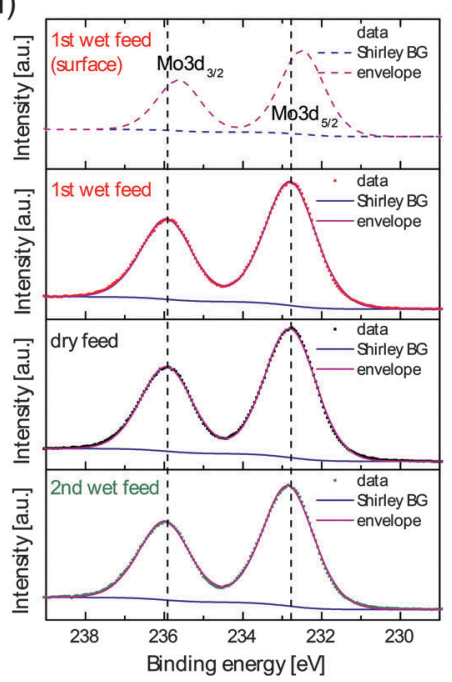

Fig. 2 Surface sensitively recorded O1s and V2p (a), V2 $p_{3 / 2}$ (b), and Mo3d (c), and bulk sensitively recorded O1s and V2p (d), V2p $3 / 2$ (e) and Mo3d (f) core level spectra (always compared to a surface spectrum in the first row) in dry and wet propane oxidation feeds.

surface sensitive investigations. The maxima of the bulk sensitively measured core level spectra of O1s, V2p, Mo3d and also Te3d (Fig. S2, ESI $\dagger$ ) are shifted by about $0.6 \mathrm{eV}$ to higher binding energies. This trend shows clearly that the core levels measured in a deeper region of the catalyst are systematically shifted to higher binding energies, meaning that the difference between the respective core and the Fermi level (at $0 \mathrm{eV}$ binding energy per definition) increases with increasing probing depth. This effect can have two possible reasons. First, the core level shifts could be caused by charging of the surface by the X-ray beam. ${ }^{28}$ The photon intensity increases with increasing photon energy at the ISISS beam line. Thus a possible charging of the catalyst surface is more pronounced at higher photon energies. However, charging affects the peak shape of the core levels inducing asymmetries. Since the Mo3d spectra shown in Fig. 2c and $\mathrm{f}$, which can be fitted with one component, are highly symmetric, charging can be excluded as explanation. Another possible reason for the binding energy shift is an upward bending of the valence and conduction bands at the M1 catalyst surface.
In the case of the n-type semiconducting M1 phase the upward band bending is induced by the formation of an electron-depleted space charge region (depletion layer). The surface of M1 is indeed more oxidized than the bulk as was proven previously. ${ }^{7,8,10,12}$ This oxidized surface provides electron acceptor states which act as traps for mobile electrons in the bulk and obviously cause a positively charged depletion layer extending into the bulk. It should also be mentioned that in XPS a surface photovoltage can arise due to the excitation of electrons by X-rays to higher states (final state effect). ${ }^{29}$ The induced electron and hole charge carriers are separated due to the band bending, i.e. electrons are accelerated into the bulk and holes to the surface, the electric field within the space charge region is compensated, and the upward bending of the bands is decreased. The same circumstance can also be present in our study - even though the applied high temperature and the usage of a polycrystalline sample at a usually high electron-hole recombination rate should make it rather unlikely - but does not influence the conclusion, since it would only decrease the initial binding energy shifts. 
Consequently, under propane oxidation conditions a depletion layer is formed proving the semiconducting response of the catalyst with charge transfer between the surface and the bulk under working conditions. However, this depletion layer or upward band bending is not influenced further by steam. This indicates that the absolute number of charge carriers in the catalyst is not affected by the presence of water in the gas phase.

\subsection{Valence band spectra and secondary electron cutoff (work function measurements)}

Since steam does not influence the band bending, its influence on the valence band and the work function was elucidated. Changes in the valence band structure can denote modified bonding motifs of the surface metal oxide moieties, while a change in the work function would directly indicate a modification of the dipole moments and hence polarity of the surface. Valence band spectra of M1 in wet and dry feeds are shown in Fig. 3. The absolute intensities of the valence band measured in the wet feed were increased by a factor of two in comparison to the dry feed. Interestingly, this effect was also observed in ultra-high vacuum valence band studies of transition metal oxides investigating the effect of chemisorbed water, ${ }^{30,31}$ though the reason remained unclear.

For a better comparison all valence band spectra depicted in Fig. 3 were normalized to their maximum. As a result, no binding energy shifts of the valence band edge dependent on steam are observed. This is in agreement with the non-shifted core level energies, hence definitely excluding the effect of water on the subsurface depletion layer and band bending. Besides, a clear difference between the relative contributions of the different components to the valence band is observed upon the addition of steam (bottom of Fig. 3). The relative spectral intensity between 4 and $8 \mathrm{eV}$ is clearly enhanced in the wet feed. An increased intensity with steam is also measured above the

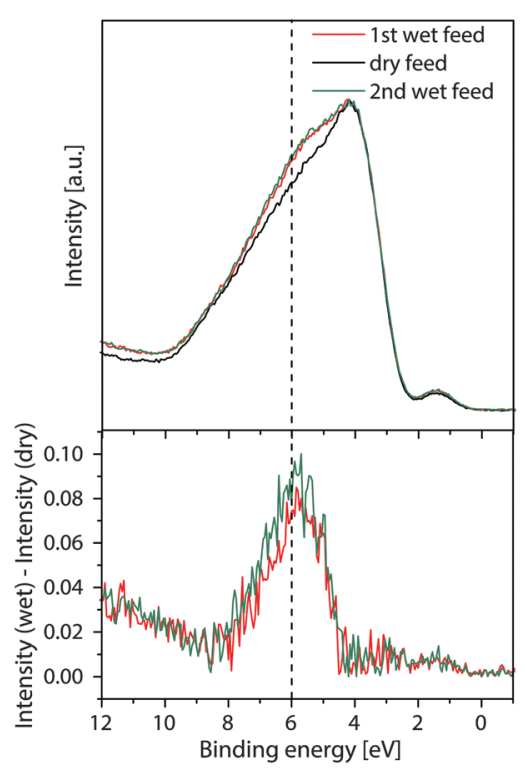

Fig. 3 Valence band spectra (normalized to their maximum) and difference spectra between wet and dry feeds recorded at $100 \mathrm{eV}$ photon energy.

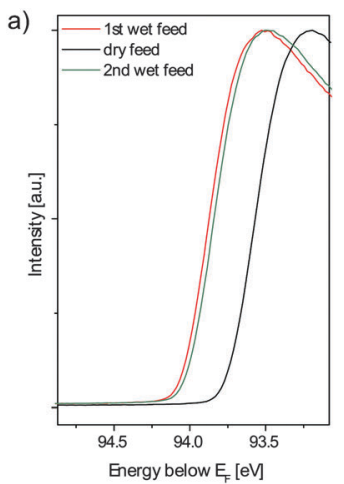

b)

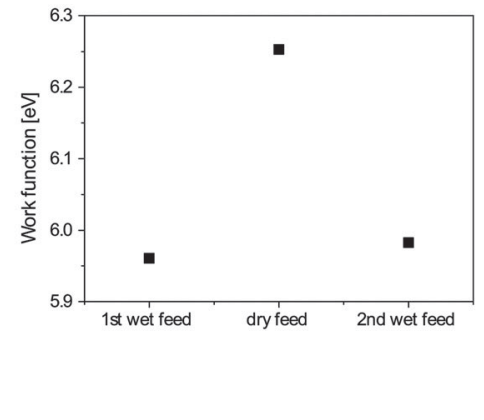

Fig. 4 (a) Secondary electron cutoff and (b) calculated work function of $\mathrm{M} 1$ in dry and wet propane oxidation feeds.

binding energy of $10 \mathrm{eV}$. The feature at $1.5 \mathrm{eV}$ is basically not affected relative to the maximum of the valence band. The assignment of different contributions to the valence band will be discussed in the resonant photoelectron spectroscopy section.

The secondary electron cutoff of the photoelectron spectrum of M1 in wet and dry feeds recorded with an excitation energy of $100 \mathrm{eV}$ is shown in Fig. 4a. The cutoff shifts reversibly to higher binding energies upon the addition of water to the feed. With the known energy position of the cutoff $E_{\text {cutoff }}$, measured at half maximum of the leading edge, and the photon energy $E_{\hbar \omega}$, the work function $\Phi$ can be calculated: ${ }^{23}$

$$
\Phi=E_{\hbar \omega}-E_{\text {cutoff }}
$$

The work function of M1 in the first wet feed is $6.0 \mathrm{eV}$, in the dry feed $6.3 \mathrm{eV}$ and in the second wet feed again $6.0 \mathrm{eV}$ (Fig. 4b). Thus the work function of M1 is reversibly decreased in the steam containing mixture. In a previous study the work function of M1 probed under similar dry feed conditions was about $6.6 \mathrm{eV} .^{12} \mathrm{~A}$ possible reason for the deviation of the absolute values in dry feed between the two experiments could be a slightly different surface roughness ${ }^{32}$ of the investigated pressed powder pellets. It is well known that the determination of precise absolute work functions even for single crystals is very difficult, since they depend very sensitively on the sample surface. ${ }^{33}$ In either case, the determined values in dry feed (6.3-6.6 eV) are only slightly lower than the work functions of $\mathrm{V}_{2} \mathrm{O}_{5}(7.0 \mathrm{eV})^{34}$ and $\mathrm{MoO}_{3}(6.9 \mathrm{eV})^{35}$ single crystals and hence are physically plausible. Moreover, during the measurement of one sample but in different gas mixtures the accuracy of the measurement is much higher (about $50 \mathrm{meV}$ ) and the reversible change by $0.3 \mathrm{eV}$ between dry and wet feeds is significant.

Work function changes can originate from a modification of the band bending and/or of the surface dipolar structure, i.e. by a change in the surface electron affinity. ${ }^{26}$ Since the effect of steam on band bending can be excluded based on the valence and core level spectra, the work function change results obviously from modified surface dipoles. As reported previously, steam significantly changes the surface composition of M1.,8,10 While the relative metal abundance of vanadium(v) (from 5 to 15\%) and tellurium (from 16 to $25 \%$ ) increases, a decrease in molybdenum 
from 55 to $35 \%$ is observed. It is therefore comprehensible that this drastic compositional change might impact the dipole momenta on the surface and hence changes also the work function of the catalyst. However, a decrease of the work function upon the adsorption of water was also observed in ultraviolet photoelectron spectroscopy studies of the binary oxides $\mathrm{V}_{2} \mathrm{O}_{3}{ }^{30}$ and $\alpha-\mathrm{Fe}_{2} \mathrm{O}_{3}{ }^{31}$ Hence also the formation of, e.g., $\mathrm{OH}$ groups that can neutralize polar surfaces, and/or probably trigger a surface reconstruction due to the reversible formation of metal hydroxides (e.g. of molybdenum), could give rise to the observed steam-induced reduction of the work function.

Summing up, steam does not change the charge carrier density of conduction electrons at the surface and subsurface of M1 since the band bending is not influenced. However, it modifies reversibly the valence band structure and surface polarity of the catalyst. In situ NEXAFS and resonant photoelectron spectroscopy were therefore performed to investigate these findings in more detail.

\subsection{Near-edge X-ray absorption fine structure (NEXAFS) spectroscopy}

The influence of steam on the electronic structure of M1 was further investigated by near-ambient pressure NEXAFS experiments. In Fig. 5a the vanadium $\mathrm{L}_{3}$-edge spectrum of $\mathrm{M} 1$ recorded in the total electron yield (TEY) mode is shown under dry and wet propane oxidation conditions at $400{ }^{\circ} \mathrm{C}$. For a better comparison, the linear-background corrected spectra were normalized to their maximum. Six prominent features can be identified. The intensities of features I $(514.9 \mathrm{eV})$ and II $(515.5 \mathrm{eV})$ decrease under wet feed conditions, while features III $(516.0 \mathrm{eV})$ and IV $(516.4 \mathrm{eV})$ remain unaffected. Features V (dry: $517.4 \mathrm{eV}$, wet: $517.6 \mathrm{eV}$ ) and VI (dry: $517.9 \mathrm{eV}$, wet: $518.2 \mathrm{eV}$ ) are reversibly shifted by $0.2-0.3 \mathrm{eV}$ to higher photon energies in the steam containing feed. a)

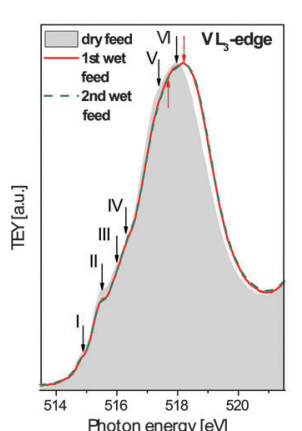

c)
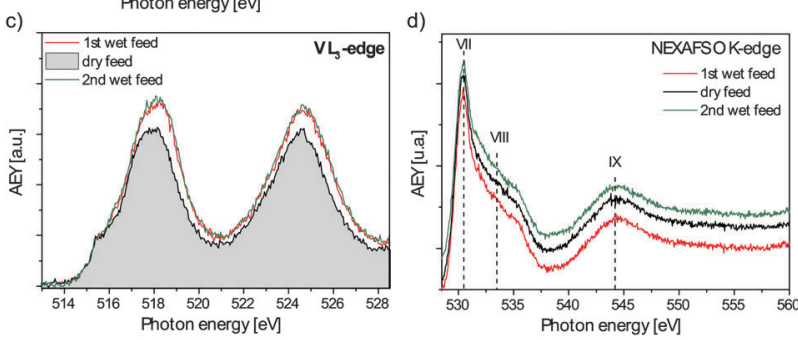

Fig. 5 (a) Vanadium $L_{3}$-edge (TEY mode), (b) 1st momentum of the vanadium $\mathrm{L}_{3}$-edge, (c) vanadium $\mathrm{L}_{2,3}$-edges (AMEY mode), and (d) oxygen $\mathrm{K}$-edge in both wet and dry feeds (stacked plot with a constant off-set).
Moreover, the relative intensity of feature $\mathrm{V}$ is decreased in the wet feed and can then only be identified as a shoulder on the low energy side of the maximum.

It could be observed that energetic shifts in the spectral weight of the $\mathrm{V} \mathrm{L}_{3}$-edge spectrum correlate with the average vanadium oxidation state of the material. ${ }^{36}$ This relationship can be understood in terms of the effective nuclear potential which affects the position of the electronic states relative to the vacuum level and the relative position of the corresponding core level. ${ }^{37}$ In the case of vanadium in the oxidation state $5+$ the effective nuclear potential is larger than that for vanadium $4+$. Thus, the vanadium(v) $\mathrm{L}_{3}$-edge is shifted to higher photon energies in comparison to the spectrum of a vanadium(Iv) or (III) compound. For a more quantitative discussion of such changes, the 1st momentum of the spectral distribution at the $\mathrm{V} \mathrm{L}_{3}$-edge can be calculated in order to precisely determine the spectral weight as a function of the gas composition. ${ }^{36}$ The 1 st momentum $M$ is calculated as

$$
M=1 / A \int \hbar \omega \mathrm{TEY} \mathrm{d} \hbar \omega,
$$

with $\hbar \omega$ being the photon energy, $A$ the integral of the vanadium $\mathrm{L}_{3}$-edge spectrum and TEY the total electron yield signal. The results of this calculation for M1 in dry and wet feeds are depicted in Fig. 5b. A reversible shift by $0.08 \mathrm{eV}$ to higher photon energies upon steam addition can be observed. This shift suggests an increase of the average vanadium oxidation state with steam, which is in agreement with the NAP-XPS experiments ( $c f$. Fig. 2b).

The bulk crystal phase M1 is composed of vanadium atoms with formal oxidation states of $4+$ and $5+$ in an octahedral coordination sphere. ${ }^{2,4}$ According to density functional theory/ restricted open shell configuration interaction single (DFT/ROCIS) calculations of $\mathrm{V}^{4+}$ and $\mathrm{V}^{5+}$ in distorted octahedral oxygen ligand fields, the low photon energy onset of the vanadium $\mathrm{L}_{3}$-edge consists of transitions into $3 \mathrm{~d}_{x y, x z}$ (in $\mathrm{V}^{5+}$ also $3 \mathrm{~d}_{y z}$ ) orbitals, ${ }^{38,39}$ which could correspond to features I and II in the experimental spectrum (Fig. 5a). These orbitals are located between $\left(3 d_{x y}\right)$ or above and below $\left(3 \mathrm{~d}_{x z}, 3 \mathrm{~d}_{y z}\right)$ the $\mathrm{V}-\mathrm{O}-\mathrm{V}$ bridging bonds in the basal plane of the $[\mathrm{VO}]_{6}$ coordination polyhedra. Thus the decreased intensity of features I and II under wet feed conditions could be assigned to a structural modification of the $3 \mathrm{~d}_{x y}$, $3 \mathrm{~d}_{x z}$, and $3 \mathrm{~d}_{y z}$ orbitals, but a clear assignment to concrete bonding motifs in M1 is difficult. While according to the calculation the main peak of $\mathrm{a} \mathrm{V}^{4+} \mathrm{L}_{3}$-edge contains transitions into $3 \mathrm{~d}_{y z}, 3 \mathrm{~d}_{x y}, 3 \mathrm{~d}_{x_{2}-y_{2}}$ and $3 \mathrm{~d}_{z_{2}}$ orbitals, it consists for $\mathrm{V}^{5+}$ species mainly of transitions into $3 \mathrm{~d}_{x_{2}-y_{2}}$, while the contribution of $3 \mathrm{~d}_{z_{2}}$ is mainly found at the high energy onset. Hence a predominant shift upon a change in the oxidation state of vanadium from $4+$ to $5+$ can be observed for the transition into $3 \mathrm{~d}_{z_{2}}$. Thus a large shift of feature $\mathrm{V}$ to higher energies can be likely assigned to the $3 \mathrm{~d}_{z_{2}}$ transition and hence to an increase of the vanadium(v) concentration on the surface. Since an increase in energy for the transition into the $3 \mathrm{~d}_{z_{2}}$ orbital is correlated with the shortening of the vanadyl bond in a distorted octahedral oxygen ligand field, ${ }^{38,39}$ steam obviously affects basically the $\mathrm{V}=\mathrm{O}$ bond motif on the surface of M1. 
Previous XPS measurements of M1 indicated that not only the average oxidation state, but also the total vanadium content on the surface increases in steam-containing feeds. ${ }^{8}$ However, the aforementioned TEY NEXAFS mode cannot be used for a comparison of absolute signal intensities in different feeds due to secondary electrons created by electron impact ionisation of gas phase molecules ("environmental electrons") that contribute strongly to the TEY signal and are hence gas phase dependent. ${ }^{40}$ In Fig. 5 c vanadium $\mathrm{L}_{2,3}$-edge spectra recorded in the Auger-Meitner electron yield (AMEY) mode, normalized to the synchrotron ring current and linear-background corrected to the intensity value at $512 \mathrm{eV}$, are shown under wet and dry feed conditions in order to compare the absolute resonance intensities. In the AMEY mode Auger-Meitner electrons with a high kinetic energy are detected, which are less strongly scattered in the gas phase. The analyzer was set to a kinetic energy of $385 \mathrm{eV}$ to suppress contributions of the gas phase (mainly $\mathrm{O}_{2}$ ) to the signal. ${ }^{21}$ In the wet feed the intensity of the vanadium $\mathrm{L}_{2,3}$-edge increases reversibly in comparison to the dry feed conditions. Assuming that the electron scattering and cascading processes in the M1 solid phase are hardly affected by the chemical modification of the surface due to the wet feed conditions, the AMEY intensity of the vanadium $\mathrm{L}_{2,3}$-edge should be proportional to the number of absorption centers. Even if the gas phase still disturbs the absolute measurement, since in the wet feed helium is replaced by steam which should induce an increase in photon absorption and inelastic photoelectron scattering, the AMEY signal would then have to decrease with steam. Since the addition of water leads to an increased signal instead, the vanadium content is obviously indeed enriched at the surface, hence supporting the XPS results.

In Fig. 5d a stacked plot of the oxygen K-edge measured also in the AMEY mode is shown. In all spectra three dominant features can be identified. Feature VII is located at $531 \mathrm{eV}$, a shoulder (feature VIII) can be found between 532 and $536 \mathrm{eV}$ and a further feature IX is identified at about $545 \mathrm{eV}$. No change in the shape of the spectra due to the addition of water to the feed can be observed. The oxygen K-edge of M1 is comparable with the oxygen K-edges of $\mathrm{MoO}_{3}{ }^{41}$ and $\mathrm{V}_{2} \mathrm{O}_{5} \cdot{ }^{42} \mathrm{MoO}_{3}$ also has three features which nearly coincide, with respect to their positions and shape, with the identified features in M1. Features VII and VIII can also be observed in $\mathrm{V}_{2} \mathrm{O}_{5}$, in contrast to feature IX which differs with respect to its position and shape. The main metal component in M1 is molybdenum. Its coordination to oxygen is similar to that observed in $\mathrm{MoO}_{3}$. In both oxides molybdenum is coordinated by six oxygen atoms in a distorted octahedral ligand field. In this context features VII ( $\pi^{*}$ resonance) and VIII ( $\sigma^{*}$ resonance) can be assigned to transitions into orbitals with the antibonding Mo4d-O2p character indicating covalent bonds between molybdenum and oxygen atoms, ${ }^{41}$ while feature IX is attributed to transitions into antibonding orbitals with Mo5s-O2p character. ${ }^{41}$ In a pure ionic oxide, the oxygen $2 \mathrm{p}$ orbitals are completely filled and no resonance occurs. Thus the oxygen K-edge dominated by contributions from molybdenumoxygen bonds indicates that molybdenum to a large extent is covalently bonded in M1.

\subsection{Resonant photoelectron spectroscopy (resPES) at the vanadium $L_{2,3}$-edge}

In order to investigate the electronic structure of the valence band in detail, resPES is the method of choice. In resPES contributions of a particular element to the valence band can be enhanced by selective excitation, ${ }^{43}$ if the normal photoelectron channel interferes with a second, element-specific excitation channel. In the case of vanadium excited at the V2p threshold both processes can be described in the following way: ${ }^{42}$

$$
\begin{gathered}
2 \mathrm{p}^{6} 3 \mathrm{~d}^{n}+\hbar \omega \rightarrow 2 \mathrm{p}^{6} 3 \mathrm{~d}^{n-1}+e^{-} \\
2 \mathrm{p}^{6} 3 \mathrm{~d}^{n}+\hbar \omega \rightarrow\left[2 \mathrm{p}^{5} 3 \mathrm{~d}^{n+1}\right]^{*} \rightarrow 2 \mathrm{p}^{6} 3 \mathrm{~d}^{n-1}+e^{-}
\end{gathered}
$$

In the second excitation process (eqn (8)) a $2 \mathrm{p}$ electron is excited to an intermediate state marked by the asterisk which is the final state in NEXAFS spectroscopy and decays by the Coster-Kronig or super-Coster-Kronig process. ${ }^{43}$ Thus the final state of the direct PES (eqn (7); described by the amplitude A) and the second excitation channel (described by the amplitude B) is the same and they can interfere:

$$
|A+B|^{2}=|A|^{2}+|B|^{2}+\text { cross term }
$$

In the case of different final states, the cross term in eqn (9) vanishes and no enhancement of the PES signal is observed.

The valence band of M1 was recorded at photon energies corresponding to the vanadium $\mathrm{L}_{2,3}$-absorption edge (Fig. 6a). To check whether the enhancement is due to a resPES process or just an overlap (incoherent enhancement) of the valence band with the Auger-Meitner decay channel, the valence band recorded at different photon energies has been plotted versus the kinetic energy of the photoelectrons. The O KLL AugerMeitner emission spectra, which could in principal overlap with the valence band, are shown in Fig. $6 \mathrm{~b}$. In the case of a non-resonant overlap, the apparent enhancement appears at constant kinetic energies. This is however not observed in our experiment (Fig. 6c). The valence band spectra, normalized to the ring current, plotted versus the binding energy are shown in Fig. 6d. Since the onsets of the valence band spectra coincide, no background correction of the spectra was necessary. In all spectra the off-resonance valence band spectrum recorded at $512 \mathrm{eV}$ is additionally plotted (dashed red line) to highlight the resonance enhancement. It can be clearly observed that features at binding energies of $1.5 \mathrm{eV}$ and in the range of $6-10 \mathrm{eV}$ are enhanced, if they are excited at photon energies corresponding to the maxima of the vanadium $\mathrm{L}_{2,3}$-edge. A plot of the valence band intensities at 1.5 and $6 \mathrm{eV}$ versus the applied photon energy is shown in Fig. 6e. As a result, the excitation energy dependence of the enhancement of the valence band spectrum follows exactly the vanadium $\mathrm{L}_{3}$ - and $\mathrm{L}_{2}$-edge spectra with maxima at 517.9 and $524.5 \mathrm{eV}$, respectively.

Zimmerman et al. performed a resPES study of $\mathrm{V}_{2} \mathrm{O}_{3}$ and $\mathrm{V}_{2} \mathrm{O}_{5} \cdot{ }^{42}$ In their investigation they identified a resonant enhancement for $\mathrm{V}_{2} \mathrm{O}_{3}$ at $1 \mathrm{eV}$ binding energy which they attributed to an occupied vanadium $3 d$ state with the oxygen $2 p$ admixture. Additionally, they found an enhancement of above $6 \mathrm{eV}$, which was also observed for $\mathrm{V}_{2} \mathrm{O}_{5}$. In an ionic bonding model of 
a)

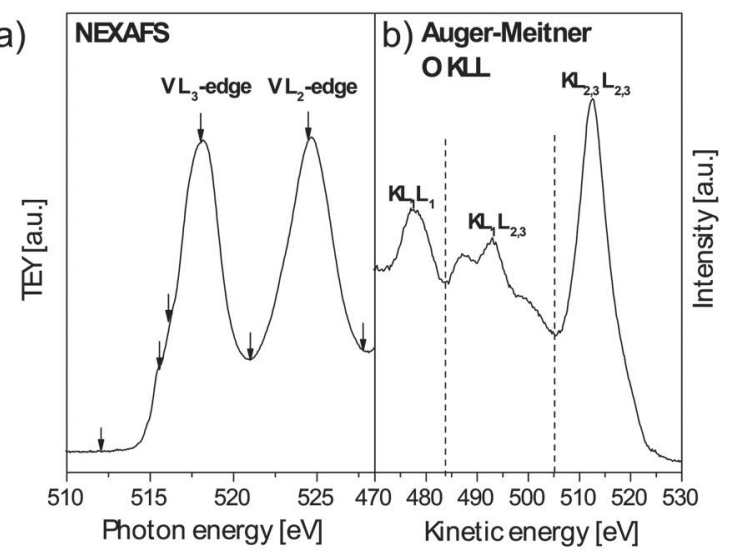

c)
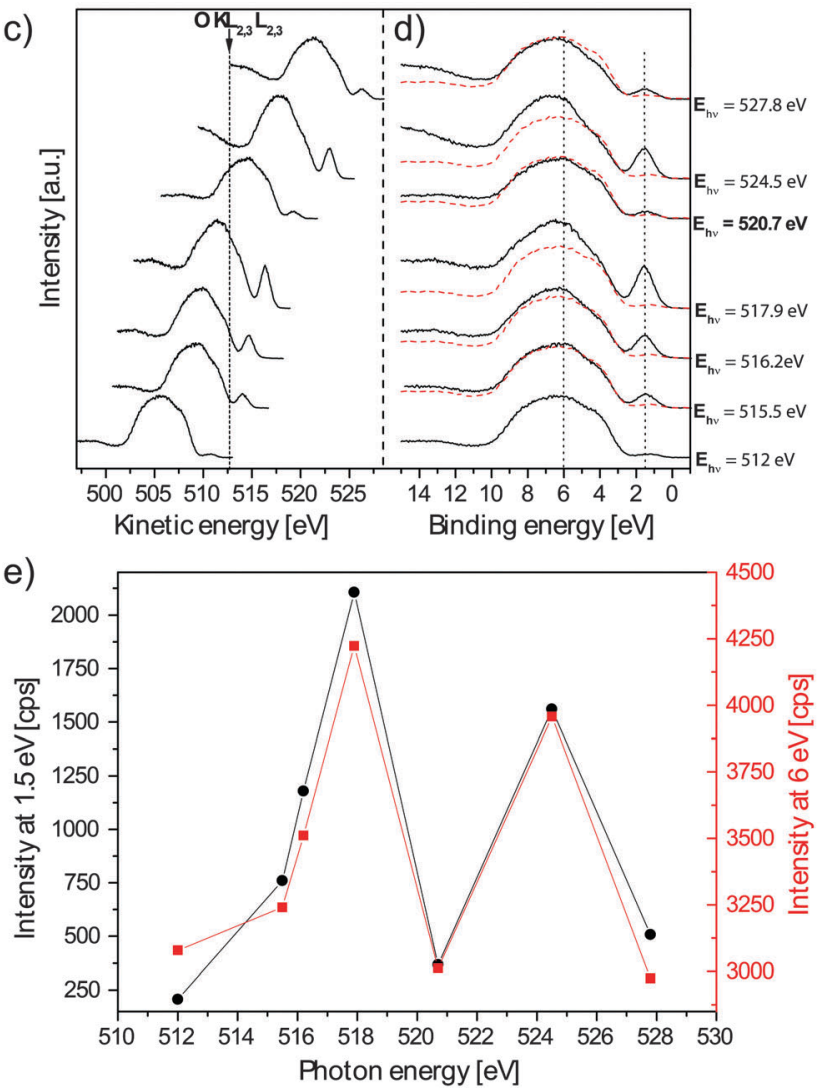

Fig. 6 (a) Vanadium $L_{2,3}$-edge with marked (arrows) photon energies at which the resPES spectra were recorded. (b) Oxygen KLL Auger-Meitner spectrum, (c) plot of resPES valence band spectra versus kinetic energy, (d) resPES valence band spectra versus binding energy, (e) intensity of the valence band at 1.5 and $6 \mathrm{eV}$ binding energies versus excitation photon energy.

binary vanadium oxides the valence band is composed of occupied $\mathrm{O} 2 \mathrm{p}$ states, while the conduction band is formed by unoccupied V3d states. In contrast, in a covalent model allowing a hybridization of V3d with $\mathrm{O} 2 \mathrm{p}$ states, the valence band has O2p and V3d character. Thus even $\mathrm{V}_{2} \mathrm{O}_{5}$, without $3 \mathrm{~d}$ valence electrons in an ionic model, contains partially occupied V3d (or mixed V3d/O2p) states. ${ }^{42,44,45}$ Zimmermann et al. attributed the enhancement above $6 \mathrm{eV}$ to the admixture of V3d states to the $\mathrm{O} 2 \mathrm{p}$ valence band suggesting a strong covalent $\mathrm{V}-\mathrm{O}$ bonding character in $\mathrm{V}_{2} \mathrm{O}_{5} \cdot{ }^{42}$ Their conclusion is supported by DFT calculations ${ }^{42,44}$ and was also seen in resPES measurements at the V3p threshold. ${ }^{46,47}$

In M1 the situation is more complicated because besides vanadium, it also contains molybdenum, tellurium and niobium atoms. However, vanadium is coordinated by oxygen in a distorted octahedron as in $\mathrm{V}_{2} \mathrm{O}_{5}$ or $\mathrm{V}_{2} \mathrm{O}_{3}$. Thus the conclusion drawn for the resPES studies of $\mathrm{V}_{2} \mathrm{O}_{3}$ and $\mathrm{V}_{2} \mathrm{O}_{5}$ can be used to interpret the resonance spectra of M1 at the vanadium $2 \mathrm{p}$ threshold. Consequently, the enhancement at $1.5 \mathrm{eV}$ binding energy in M1 can be assigned to a partially occupied V3d state. The enhancement between the binding energies 6 and $10 \mathrm{eV}$ can be interpreted in terms of hybridization of V3d with O2p states similar to the results obtained for $\mathrm{V}_{2} \mathrm{O}_{3}$ and $\mathrm{V}_{2} \mathrm{O}_{5}$. Thus vanadium shows also in $\mathrm{M} 1$ a strong covalent bonding character. These results are in agreement with the interpretation of the oxygen K-edge spectra with $\sigma^{*}$ and $\pi^{*}$ resonance components (Fig. 5d), which is mainly attributed to the hybridization between molybdenum $4 \mathrm{~d}$ and oxygen $2 \mathrm{p}$ orbitals and hence points to a strong covalent molybdenum-oxygen bonding character.

As discussed before, steam induced a significant relative increase of the signal intensity at binding energies in the $6-10 \mathrm{eV}$ range of the valence band (Fig. 3). This can now be interpreted as an enhancement of the vanadium $3 \mathrm{~d}$ state admixture to the valence band. This observation agrees with the results obtained from NEXAFS AMEY mode measurements at the $\mathrm{L}_{2,3}$-edge (Fig. 5c) and from NAP-XPS results, ${ }^{7,10}$ where an increased vanadium abundance on the surface was observed upon the addition of water to the feed. In the studies on the adsorption of water on metal oxides, peaks in the valence band spectrum between 6 and $12 \mathrm{eV}$ were assigned to undissociated, strongly chemisorbed, and dissociated water. ${ }^{48,49}$ For example, for ceria on platinum, signals at 10.8 and $7.6 \mathrm{eV}$ were assigned to the $3 \mathrm{~s}$ and $1 \mathrm{p}$ molecular orbitals of hydroxyl groups. ${ }^{49}$ Consequently, the increased emission above $10 \mathrm{eV}$ observed for the M1 phase might also arise from the formation of $\mathrm{OH}$ groups on the surface of the catalyst upon dissociative adsorption of water. However, in $\mathrm{V}_{2} \mathrm{O}_{5}$ a very broad satellite of the valence band, which is also seen in other transition metal oxides occurs between the valence band and the oxygen $2 \mathrm{~s}$ level above $10 \mathrm{eV}^{47,50}$ If a feature is enhanced in the valence band, the corresponding satellite is also enhanced because the main line(s) (valence band) and the satellite belong to the same ground state(s). Thus V3d states could also contribute to the increased intensity above $10 \mathrm{eV}$ upon steam addition.

Since the peak at $1.5 \mathrm{eV}$ binding energy can now be assigned to a (partially) filled vanadium $3 \mathrm{~d}$ state, steam obviosly does not influence its occupation (Fig. 3). Interestingly, it is known from NAP-XPS studies that only the $\mathrm{V}^{5+}$ concentration increases upon steam addition, while the $\mathrm{V}^{4+}$ concentration remains constant. ${ }^{7,8,10}$ The constant and not steam-influenced intensity at $1.5 \mathrm{eV}$ representing basically vanadium with $3 \mathrm{~d}$ electrons such as $\mathrm{V}^{4+}$ is therefore in good agreement with these findings, while the enhancement of the valence band at $6-10 \mathrm{eV}$ might be due to surface-enriched, covalently bonded $\mathrm{V}^{5+}$ species.

A changed valence (and likely conduction band) structure also means that the dispersion relation of the energy band and 
therefore the effective mass of the charge carriers is modified. According to the Drude-Lorentz model used to describe charge carrier dynamics in semiconductors, ${ }^{51}$ a modified effective mass $m^{*}$ changes the plasmon frequency $\omega_{\mathrm{p}}=n_{\mathrm{e}} e^{2} /\left(\varepsilon_{0} m^{*}\right)$ (with $n_{e}$ being the charge carrier density of conduction electrons, $e$ the elementary charge and $\varepsilon_{0}$ the vacuum permittivity) and therefore the mobility of the charge carriers $\mu_{\mathrm{e}}=e^{2} \tau / m^{*}$ (with $\tau$ being the relaxation time). This affects finally the conductivity as shown by eqn (4). Thus the modification of the effective mass and hence electron mobility would explain the decreased conductivity in a steam containing propane oxidation feed ( $c f$. Fig. 1a). And furthermore, a higher covalency will also decrease the dipole moment in a metal oxide and consequently its work function, in agreement with the experimental observations.

\section{Conclusions}

The selective propane oxidation catalyst $\mathrm{MoVTeNbO}_{x}$ (orthorhombic M1 phase) was investigated by microwave conductivity, synchrotron X-ray photoelectron, soft X-ray absorption and resonant photoelectron spectroscopy under reaction conditions to identify the influence of steam on its bulk and surface electronic properties. The investigation of steam-induced structural modifications might help us to understand the observed steam-induced increase of the selectivity to the target product acrylic acid. We found that steam decreases the electrical conductivity, increases the concentration of $\mathrm{V}^{5+}$ and $\mathrm{Te}^{6+}$ and decreases the relative amount of $\mathrm{Mo}^{6+}$ on the surface, decreases the work function and modifies the valence band structure. Furthermore, we could observe that the catalyst forms a subsurface space charge region depleted in electrons (upward band bending) under reaction conditions. This proves that the catalyst acts like a semiconducting gas sensor (chemiresistor), where the (bulk) conductivity can be used to sensor electronic changes on the surface. However, since the addition of steam to the reaction feed does not affect the extent of band bending, it obviously does not influence the number of charge carriers in the space charge region by an additional bulk surface charge transfer. This could mean that bulk charge carriers do not participate in the formation of acrylic acid. The presence of band bending and hence the formation of a high surface potential barrier, which electrons have to overcome on their way from the bulk to the surface, could be however necessary to terminate the bulk surface charge transfer, limit the formation of active oxygen species on the surface and to achieve finally the high selectivity to acrylic acid, while suppressing its total oxidation to $\mathrm{CO}_{2}$. The surface potential barrier and the limited bulk surface charge transfer would also explain the extraordinary stability of the $\mathrm{MoVTeNbO}_{x}$ M1 bulk phase under reaction conditions.

However, steam modifies strongly the electronic and chemical structure of the surface. $\mathrm{V} \mathrm{L}_{3}$-edge NEXAFS experiments point to a particular modification of the vanadyl bond motif $(\mathrm{V}=\mathrm{O})$ on the surface and changes in the valence band spectra suggest an enrichment of covalently bonded $\mathrm{V}^{5+}$ surface species. The increased covalent bonding character in the multi-metal oxide and the decreased work function indicating a decreased surface polarity might induce a decrease of the charge carrier mobility. This effect would hence explain the observed decreased conductivity in the steam-containing reaction feed. These results point to the conclusion that steam is basically needed to form the active surface layer that can selectively oxidize propane to acrylic acid.

\section{Acknowledgements}

We thank Dr Maricruz Sanchez-Sanchez for the synthesis of the MoVTeNb oxide M1 sample. The HZB staff is acknowledged for their continual support of the high-pressure electron spectroscopy activities of the FHI at BESSY II.

\section{References}

1 F. Cavani, Catal. Today, 2010, 157, 8-15.

2 P. DeSanto Jr, D. J. Buttrey, R. Grasselli, W. Pyrz, C. Lugmair, A. F. Volpe Jr, T. Vogt and B. Toby, Top. Catal., 2006, 38, 31-40.

3 Y. Moro-oka, W. Ueda and K.-H. Lee, J. Mol. Catal. A: Chem., 2003, 199, 139-148.

4 H. Murayama, D. Vitry, W. Ueda, G. Fuchs, M. Anne and J. Dubois, Appl. Catal., A, 2007, 318, 137-142.

5 D. Vitry, J.-L. Dubois and W. Ueda, J. Mol. Catal. A: Chem., 2004, 220, 67-76.

6 W. D. Pyrz, D. A. Blom, N. R. Shiju, V. V. Guliants, T. Vogt and D. J. Buttrey, Catal. Today, 2009, 142, 320-328.

7 A. Celaya Sanfiz, T. W. Hansen, D. Teschner, P. Schnörch, F. Girgsdies, A. Trunschke, R. Schlögl, M. Hong Looi and S. B. Abd Hamid, J. Phys. Chem. C, 2010, 114, 1912-1921.

8 R. Naumann d'Alnoncourt, L.-I. Csepei, M. Hävecker, F. Girgsdies, M. E. Schuster, R. Schlögl and A. Trunschke, J. Catal., 2014, 311, 369-385.

9 R. Schlögl, Top. Catal., 2011, 54, 627-638.

10 M. Hävecker, S. Wrabetz, J. Kröhnert, L. I. Csepei, R. N. d'Alnoncourt, Y. V. Kolen'ko, F. Girgsdies, R. Schlögl and A. Trunschke, J. Catal., 2012, 285, 48-60.

11 X. Rozanska, R. Fortrie and J. Sauer, J. Phys. Chem. C, 2007, 111, 6041-6050.

12 C. Heine, M. Hävecker, M. Sanchez-Sanchez, A. Trunschke, R. Schlögl and M. Eichelbaum, J. Phys. Chem. C, 2013, 117, 26988-26997.

13 M. Eichelbaum, R. Stöer, A. Karpov, C.-K. Dobner, F. Rosowski, A. Trunschke and R. Schlögl, Phys. Chem. Chem. Phys., 2012, 14, 1302-1312.

14 J. C. Slater, Rev. Mod. Phys., 1946, 18, 441-512.

15 L.-F. Chen, C. K. Ong, C. P. Neo, V. V. Varadan and V. K. Varadan, Microwave Electronics: Measurement and Materials Characterization, Wiley, 2004.

16 H. Looyenga, Physica, 1965, 31, 401-406.

17 D. C. Dube, J. Phys. D: Appl. Phys., 1970, 3, 1648-1652.

18 C. Heine, M. Hävecker, F. Girgsdies, A. Trunschke, R. Schlögl and M. Eichelbaum, Appl. Phys. A: Mater. Sci. Process., 2013, 112, 289-296. 
19 H. Bluhm, M. Hävecker, A. Knop-Gericke, M. Kiskinova, R. Schlögl and M. Salmeron, MRS Bull., 2007, 32, 1022-1030.

20 E. M. Vass, M. Hävecker, S. Zafeiratos, D. Teschner, A. Knop-Gericke and R. Schlögl, J. Phys.: Condens. Matter, 2008, 20, 184016.

21 M. Hävecker, M. Cavalleri, R. Herbert, R. Follath, A. Knop-Gericke, C. Hess, K. Hermann and R. Schlögl, Phys. Status Solidi B, 2009, 246, 1459-1469.

22 S. Tanuma, C. J. Powell and D. R. Penn, Surf. Interface Anal., 1988, 11, 577-589.

23 M. G. Helander, M. T. Greiner, Z. B. Wang and Z. H. Lu, Appl. Surf. Sci., 2010, 256, 2602-2605.

24 G. L. Pearson and J. Bardeen, Phys. Rev., 1949, 75, 865-883.

25 C. Heine, M. Havecker, E. Stotz, F. Rosowski, A. Knop-Gericke, A. Trunschke, M. Eichelbaum and R. Schlogl, J. Phys. Chem. C, 2014, 118, 20405-20412.

26 H. Lüth, Electronic Structure of Surfaces, Solid Surfaces, Interfaces and Thin Films, Springer Verlag, 2001, pp. 265-328.

27 R. Tokarz-Sobieraj, K. Hermann, M. Witko, A. Blume, G. Mestl and R. Schlögl, Surf. Sci., 2001, 489, 107-125.

28 B. J. Tielsch and J. E. Fulghum, Surf. Interface Anal., 1996, 24, 28-33.

29 D. K. Schroder, Meas. Sci. Technol., 2001, 12, R16.

30 R. L. Kurtz and V. E. Henrich, Phys. Rev. B: Condens. Matter Mater. Phys., 1983, 28, 6699-6706.

31 R. L. Kurtz and V. E. Henrich, Phys. Rev. B: Condens. Matter Mater. Phys., 1987, 36, 3413-3421.

32 W. Li and D. Y. Li, J. Chem. Phys., 2005, 122, 064708.

33 V. E. Heinrich and P. A. Cox, The Surface Science of Metal Oxides, Univ. Pr., Cambridge, 1996.

34 J. Meyer, K. Zilberberg, T. Riedl and A. Kahn, J. Appl. Phys., 2011, 110, 033710.

35 M. T. Greiner, L. Chai, M. G. Helander, W. M. Tang and Z. H. Lu, Adv. Funct. Mater., 2012, 22, 4557-4568.

36 M. Hävecker, A. Knop-Gericke, R. W. Mayer, M. Fait, H. Bluhm and R. Schlögl, J. Electron Spectrosc. Relat. Phenom., 2002, 125, 79-87.
37 J. Chen, C. Kirn, B. Frähberger, B. Devries and M. Touvelle, Surf. Sci., 1994, 321, 145-155.

38 D. Manganas, M. Roemelt, T. Weyhermüller, R. Blume, M. Hävecker, A. Knop-Gericke, S. DeBeer, R. Schlögl and F. Neese, Phys. Chem. Chem. Phys., 2014, 16, 264-276.

39 D. Manganas, M. Roemelt, M. Hävecker, A. Trunschke, A. Knop-Gericke, R. Schlögl and F. Neese, Phys. Chem. Chem. Phys., 2013, 15, 7260-7276.

40 N. J. Shevchik and D. A. Fischer, Rev. Sci. Instrum., 1979, 50, 577-581.

41 M. Cavalleri, K. Hermann, S. Guimond, Y. Romanyshyn, H. Kuhlenbeck and H.-J. Freund, Catal. Today, 2007, 124, 21-27.

42 R. Zimmermann, R. Claessen, F. Reinert, P. Steiner and S. Hufner, J. Phys.: Condens. Matter, 1998, 10, 5697-5716.

43 N. Martensson, O. Karis and A. Nilsson, J. Electron Spectrosc. Relat. Phenom., 1999, 100, 379-393.

44 V. Eyert and K.-H. Höck, Phys. Rev. B: Condens. Matter Mater. Phys., 1998, 57, 12727-12737.

45 G. A. Sawatzky and D. Post, Phys. Rev. B: Condens. Matter Mater. Phys., 1979, 20, 1546-1555.

46 K. E. Smith and V. E. Henrich, Phys. Rev. B: Condens. Matter Mater. Phys., 1988, 38, 9571-9580.

47 S. Shin, Y. Tezuka, T. Ishii and Y. Ueda, Solid State Commun., 1993, 87, 1051-1054.

48 W. R. Flavell, A. G. Thomas, J. Hollingworth, S. Warren, S. C. Grice, P. M. Dunwoody, C. E. J. Mitchell, P. G. D. Marr, D. Teehan, S. Downes, E. A. Seddon, S. Downes, E. A. Seddon, V. R. Dhanak, K. Asai, Y. Koboyashi and N. Yamada, Faraday Discuss., 1999, 114, 407-420.

49 U. Berner, K. Schierbaum, G. Jones, P. Wincott, S. Haq and G. Thornton, Surf. Sci., 2000, 467, 201-213.

50 K. W. Goodman and V. E. Henrich, Phys. Rev. B: Condens. Matter Mater. Phys., 1994, 50, 10450-10456.

51 R. Ulbricht, E. Hendry, J. Shan, T. F. Heinz and M. Bonn, Rev. Mod. Phys., 2011, 83, 543-586. 